\title{
ASPECTOS DO AUTOCUIDADO EM SAÚDE BUCAL DE IDOSOS HIPERTENSOS E DIABÉTICOS QUE VIVEM SOZINHOS
}

\author{
ORAL SELF-CARE OF HYPERTENSIVE AND DIABETIC SENIORS
}

\section{Patrícia de Lima ${ }^{a}$, Ananyr Porto Fajardo ${ }^{b}$}

\author{
apati_tkn@msn.com, baportofajardo@gmail.com
}

Grupo Hospitalar Conceição - Porto Alegre (RS), Brasil

Data de recebimento: 04/03/2016

Data de aceite do artigo: 28/09/2016

\section{RESUMO}

Introduçáo: $\mathrm{O}$ incremento no número de idosos morando sozinhos no sul do Brasil indica a necessidade de autonomia no autocuidado em saúde. Considerando a prevalência de hipertensão e diabetes nesse grupo populacional, destaca-se a maneira como cuidam da saúde bucal. Objetivos: Conhecer o autocuidado em saúde bucal de idosos hipertensos e diabéticos que vivem sozinhos. Materiais e métodos: Investigação qualitativa, exploratório-descritiva, realizada no território adscrito a uma unidade de atenção primária em saúde de Porto Alegre (RS). Oito sujeitos foram entrevistados em domicílio. Os dados sociodemográficos foram descritos e as respostas abertas foram analisadas tematicamente. Resultados e discussáo: $\mathrm{O}$ grupo era formado predominantemente por mulheres, viúvas, maiores de 70 anos e com nível de escolaridade inversamente proporcional à idade. Procuravam atendimento odontológico para problemas pontuais e usavam prótese, quando bem adaptada. As dificuldades para seu uso eram náusea, má adaptação e obstáculos para agendar seu restauro no serviço de referência. Compreendiam que hipertensão e diabetes são agravos crônicos, precisando controlá-los. Apresentavam conhecimento nutricional satisfatório, diminuição do apetite e do prazer com alimentação por limitaçóes na dieta e hipossalivaçáo, cuja ocorrência era relacionada com respiração bucal, condiçóes crônicas em questão e farmacoterapia. Usavam corretamente medicamentos indicados e compreendiam sua importância, com evidências de polifarmácia. Conclusóes: Os participantes demonstraram que cuidavam da própria saúde bucal seguindo orientaçóes prescritas e com apoio de familiares e do serviço de saúde de referência. Indicaram que assimilaram diversas orientaçóes a seu estilo de vida. Compreendiam a relação entre determinadas terapêuticas farmacológicas e incômodos bucais.

Palavras-chave: Saúde bucal; serviços de saúde para idosos; assistência odontológica para idosos; hipertensão; diabetes mellitus; autocuidado.

\section{ABSTRACT}

Introduction: The increase in the number of elderly people living alone in the south of Brazil points to the need of autonomy in health self-care. Considering the prevalence of hypertension and diabetes in this age group, the relevance of their oral self-care is highlighted. Objectives: To know the oral health self-care of hypertensive and diabetic elderly people who live alone. Material and methods: Qualitative, exploratory-descriptive research, conducted in the territory under responsibility of a primary health care service in Porto Alegre (Rio Grande do Sul State, Brazil). Eight subjects were interviewed in their homes. The socio-demographic data were described and the open answers were thematically analyzed. Results and discussion: They were predominantly women, widows, older than 70 years-old and with years of schooling inversely proportional to their age. They searched for dental care for occasional problems and used prostheses, when well adapted. The restrictions to their use were nausea, ill-adaptation and obstacles to schedule its fixing in the mentioned service. Hypertension and diabetes were understood as chronic conditions, being necessary to control them. They presented an adequate nutritional knowledge, decreased appetite and low satisfaction when eating due to diet limitation and hyposalivation, which occurrence was related to oral breathing, the mentioned chronic conditions and drug therapy. They used their medication correctly, understanding its importance and evidencing polypharmacy. Conclusions: The participants followed prescribed measures and were supported by family members and the reference health service. They indicated that they have assimilated several recommendations to their lifestyle. They understood the relationship between certain pharmacological therapies and oral discomfort.

Keywords: Oral health; health services for aged; dental care for aged; hypertension; diabetes mellitus; self-care. 


\section{INTRODUÇÃO}

O envelhecimento humano é um fenômeno mundial e requer grande atenção dos serviços e políticas públicas, visto que o padrão familiar dos idosos está mudando e há uma tendência ao incremento do número de lares compostos por uma única pessoa. A região Sul do Brasil se destaca nesses aspectos: Porto Alegre, capital do Rio Grande do Sul (RS), ocupa o primeiro lugar na proporção de idosos morando sozinhos $(27,1 \%)^{1}$.

Morar sozinho pode ser uma alternativa para idosos que lutam para manter sua independência e autonomia ou inevitável para aqueles que, apesar de se sentirem sós ou abandonados, não possuem outras pessoas com as quais possam corresidir ${ }^{2}$. No entanto, a ausência de uma companhia próxima pode dificultar o socorro em casos de emergência, sendo uma condição importante para o aumento da fragilidade e vulnerabilidade do idoso, sobretudo quando há ausência do contato familiar e isolamento social ${ }^{3}$.

Simultaneamente, testemunhamos uma transição epidemiológica. O envelhecimento promove diversas alterações fisiológicas no organismo, bem como favorece o desenvolvimento de patologias, em especial as doenças crônicas, destacando-se entre elas a Hipertensão Arterial Sistêmica (HAS) e o Diabetes Mellitus (DM). A prevalência de diabetes no Brasil para adultos maiores de 65 anos é de 22,9\%, índice bastante semelhante ao do Sul do país (22,5\%). A prevalência de hipertensão arterial é muito expressiva para essa faixa etária, representando 59,2\% dos idosos maiores de 65 anos, sendo que na regiāo Sul observa-se uma prevalência de 57,6\% ${ }^{4}$.

As condições de saúde bucal na terceira idade são fundamentais para o envelhecimento saudável e uma boa qualidade de vida, destacando-se a cárie radicular e a doença periodontal como os problemas bucais prevalentes em idosos no Brasil ${ }^{5}$. Paralelamente, o país vem apresentando índices decrescentes de edentulismo (perda parcial ou total de dentes) e, gradativamente, demanda novas necessidades de tratamento reabilitador ${ }^{6}$. Esse fato acompanha a evolução da atenção à saúde bucal e o investimento em políticas públicas de promoção de saúde e fluoretação das águas. É importante destacar que a doença periodontal é a complicação crônica relacionada ao DM mais comum devido a sua alta prevalência, havendo uma inter-relação amplamente conhecida na literatura científica entre as duas condiçóes. Observase que indivíduos diabéticos apresentam, aproximadamente, duas vezes mais prevalência e maior severidade da doença periodontal do que indivíduos sem essa alteração sistêmica. Isso pode ser explicado pelo fato de a concentração sanguínea de citocinas pró-inflamatórias e prostaglandinas estar mais elevada devido à hiperglicemia crônica ${ }^{7,8}$. Por outro lado, o estado de saúde bucal também exerce influência sobre o controle do DM. A presença de infecçóes, como a periodontite, leva à estimulação da resposta inflamatória, que resulta em situação de estresse e aumenta a resistência dos tecidos à insulina, o que provoca a piora do controle glicêmico ${ }^{6}$. Sendo assim, o diabetes pode ser considerado como modificador ou fator de risco para periodontite, assim como a doença periodontal demonstra ser fator de risco significativo para doença cardiovascular decorrente de DM, chegando a representar um aumento de 2,3 vezes na prevalência de doença cardíaca isquêmica e 8,5 vezes maior para nefropatia diabética em pacientes acometidos por doença periodontal ${ }^{8}$.

Em relação à hipertensão arterial sistêmica, não está claramente estabelecido na literatura qual é o valor de pressão arterial (PA) seguro para a realização de um procedimento odontológico de urgência. Porém, na prática clínica, adotam-se os valores de PA até $180 / 110 \mathrm{mmHg}$ como valores limites ${ }^{9}$. Deve-se ter cuidado com o risco de endocardite bacteriana para o paciente que apresente cardiopatia associada. Em procedimentos invasivos, dependendo do caso, é indicada então a quimioprofilaxia antibiótica (amoxicilina como droga de eleição) para endocardite?

Até o momento, não há consenso comprovando que o emprego de vasoconstritores, respeitando as dosagens seguras, cause alteraçóes cardiovasculares. No entanto, quando o paciente apresenta complicaçóes vasculares e cardíacas, deve-se estar atento para o uso de anestésicos locais. São consideradas contraindicaçóes absolutas para o uso de vasoconstritores adrenérgicos: angina instável, ocorrência recente ( $<6$ meses) de acidente vascular cerebral (AVC) ou infarto do miocárdio, arritmias refratárias, HAS grave não controlada, insuficiência cardíaca congestiva não controlada e DM não controlado ${ }^{10}$.

$\mathrm{O}$ autocuidado pode ser descrito como processo de busca das potencialidades do indivíduo no cuidado de si, identificando os fatores que influenciam comportamentos saudáveis a partir do contexto biopsicossocial. Com isso, amplia-se o campo de possibilidades e a autonomia do próprio sujeito para optar por seus comportamentos e preferências sem basear-se exclusivamente nas percepções do profissional ${ }^{11}$.

$\mathrm{O}$ estudo aqui relatado teve como objetivo conhecer como se dá o autocuidado em saúde bucal de idosos hipertensos e diabéticos que vivem sozinhos no território adscrito a uma unidade de atenção primária à saúde localizada em Porto Alegre (RS). Especificamente, procurou-se conhecer seus hábitos de higiene oral, a dieta preparada e ingerida por esses sujeitos, correlacionar o uso dos medicamentos com efeitos sobre a saúde bucal, verificar o uso de serviços de atendimento odontológico e relacionar o autocuidado em saúde bucal com os cuidados em HAS e DM. 


\section{MATERIAIS E MÉTODOS}

A investigação qualitativa, exploratório-descritiva foi realizada no território adscrito à Unidade de Saúde Vila Floresta do Grupo Hospitalar Conceição (USVF/ GHC), localizado na zona norte de Porto Alegre (RS) e caracterizado por um número expressivo de moradores idosos. Os participantes foram entrevistados em seu domicílio, sendo a gravaçáo posteriormente transcrita e os dados submetidos à análise temática. O roteiro da entrevista incluía aspectos referentes a dados sociodemográficos, saúde bucal, alimentação e cuidados relacionados a HAS e DM. Os participantes estão identificados pela letra "P" seguida de um número de ordem aleatória que preserva o sigilo das informações prestadas.

Os dados qualitativos foram transcritos e interpretados seguindo a técnica de Análise Temática ${ }^{12}$. Os dados sociodemográficos foram descritos conforme sua frequência. A partir do Sistema de Informaçóes local, selecionaram-se indivíduos maiores de 60 anos que morassem sozinhos e apresentassem hipertensão e diabetes concomitantemente, obtendo-se o total de vinte idosos. Destes, seis recusaram-se a participar da pesquisa, três não foram encontrados após três tentativas sucessivas e três não apresentavam HAS e DM associadas concomitantemente, constituindo incorreçóes nos registros da unidade de saúde. Com isso, o universo foi composto por oito participantes.

$\mathrm{O}$ projeto de pesquisa foi submetido à apreciação do Comitê de Ética em Pesquisa do Hospital Nossa Senhora da Conceição (HNSC/GHC) - obedecendo à Resolução no 466/2012 do Conselho Nacional de Saúde, que trata de pesquisas envolvendo seres humanos - e aprovado em 09/02/2015, conforme CAAE: 39830114.6.0000.5530/CEP: 949.102/Projeto 14-259.

\section{RESULTADOS E DISCUSSÃO}

O grupo de participantes era formado predominantemente por mulheres, em estado de viuvez, acima de 70 anos de idade, com um número de filhos variando entre 0 e 9, com escolaridade equivalente ao ensino fundamental incompleto. Observa-se uma relaçáo decrescente entre a idade e o nível de escolaridade, tendo sido relatado que a origem de zona rural fez que começassem a trabalhar muito cedo e, com isso, tivessem que abandonar a escola precocemente.

É comum, entre a população idosa, a crença de que limitações relacionadas à saúde bucal façam parte de um processo natural do envelhecimento, tornando-os mais tolerantes a esses problemas. Quando questionados se havia algo incomodando na boca, todos os participantes negaram, com exceção de um. Há uma tendência em associar a palavra "incômodo" com "dor" - se não dói, não há incômodo. O fato do idoso não perceber a limitação funcional oral parece ser determinante para não sentir-se incomodado, seja por desconhecimento ou desesperança na possibilidade de atendimento odontológico, resultando em resignação com as condiçóes bucais ${ }^{13}$. Entretanto, um dos entrevistados manifestou preocupaçáo com a aparência dos dentes, demonstrando sua importância funcional e estética e não compreendendo bem o motivo de não poder recuperá-los.

P1: Não uso [prótese] porque não dá pra fazer. Eles dizem que não dá, porque tenho diabete, aí... [...] o mais que eu queria era dente de tudo. Queria, como queria... mas não deu...

Com a evolução da odontologia conservadora e das políticas públicas em saúde bucal, pode-se observar uma redução nos índices de edentulismo na população brasileira. No entanto, ainda observamos que a falta de dentes e de reabilitação protética continua a acometer a população de maneira expressiva, sendo que essa condição influencia diretamente a qualidade de vida, a autoestima, a alimentação adequada e o envelhecimento saudável.

Quando questionados sobre o uso de prótese dentária, mais da metade a utilizava e relatava estar bem adaptada. No entanto, dentre os que apresentaram queixas, relataram problemas como dificuldade de usá-la porque machuca, causa ânsia de vômito e a dificuldade em marcar atendimento e consultar na unidade de saúde.

P5: Andei indo ali no posto de saúde, mas é muito difícil para marcar consulta. E ali não é tudo que eles fazem, não é todo o tratamento.

$\mathrm{O}$ atendimento odontológico oferecido pela unidade de saúde de referência era procurado para resolver problemas pontuais, como quando algo estava incomodando ou para consertar a prótese. A maioria relatou que a última consulta ao dentista foi até três anos atrás, havendo uma pessoa que não ia à unidade há aproximadamente 18 anos, não sentindo mais necessidade de consultar por náo possuir mais dentes. $\mathrm{O}$ fato de não frequentar o serviço odontológico indica um forte componente cultural na priorização da atenção à saúde, tradicionalmente voltada à população mais jovem, podendo-se presumir que os mais velhos não buscam atendimento devido à ausência de dor ou à aceitação de uma condição há muito estabelecida, como o edentulis$\mathrm{mo}^{14}$. Em contrapartida, a dificuldade de acesso ao atendimento, a história pregressa de uma odontologia mutiladora e a incerteza da resolutividade do tratamento 
levam ao sentimento de conformismo na autopercepção em saúde bucal. Sendo assim, é comum observarmos que, para muitos idosos, a visita ao dentista é importante apenas para quem possui dentes ${ }^{13}$. Um relato indica a busca de outros recursos por iniciativa própria:

P3: Eu tive uns negócios que tavam entrando, cortando o dente, mas eu fui no dentista particular porque ali [na USVF] não faziam, e ela fechou tudo e não incomodou mais.

Em relação à higiene bucal, as pessoas afirmaram realizar escovação dos dentes e das próteses com dentifrício fluoretado. Quatro idosos faziam uso esporádico de enxaguatório bucal, dois relataram colocar as próteses em solução de água sanitária para deixá-las mais limpas e brancas e uma pessoa relatou que apenas enxaguava a boca com água.

P2: Escovo muito meus dentes. Quando posso fazer faço gargarejo. Uso pasta de dente. Tiro só pra escovar. Não fico sem elas nenhum minutinho. Não sei nem falar sem elas.

A condição de saúde da boca deve ser considerada como fator relevante no autocuidado de saúde dos indivíduos, já que problemas de saúde bucal têm repercussóes nutricionais e psicossociais vastamente relatadas na literatura científica ${ }^{14}$. A falta de conhecimento dos pacientes diabéticos em relação às complicaçôes orais do DM e a manutenção deficiente da adequada saúde bucal, por exemplo, são importantes indicadores de risco para o DM, prejudicando o controle glicêmico ${ }^{6,15}$.

Em relação à alimentação, quando questionados quem preparava as refeiçôes, sete responderam que eles mesmos o faziam, justificando que a comida deveria conter pouca gordura, pouco sal e açúcar controlado ou porque não havia outra pessoa que a preparasse. Um participante disse que sempre encomendava a refeição em viandas, outro realizava a refeição principal em um centro de convivência de idosos do território. Foi frequente o relato do consumo de frutas e lanches entre refeiçóes ao longo do dia.

De maneira geral, os relatos indicaram que a alimentação ingerida era balanceada, evidenciando conhecimento dos alimentos indicados em função de suas enfermidades. Os entrevistados expressavam um conhecimento nutricional satisfatório, procurando obedecer a restriçóes alimentares estabelecidas para se evitar exageros que contribuíam para as comorbidades apresentadas. Em vários excertos também ficou evidente o conhecimento próprio sobre saúde e a importância atribuída às orientaçóes para seu autocuidado:
P4: Me cuido muito com comida, porque a gente sabe que não pode abusar com nada quando a gente tem problema, pode ter piora da diabetes... é coisa séria. A gente tem que se cuidar, então eu cuido muito.

P5: Eu não como doce, por causa da diabetes. Porque já atingiu minhas vistas, atingiu meu rim, antes de eu saber que en era diabética.

P1: Eu me cuido, e como me cuido. Porque é ruim gente doente, né? Quero melhorar da diabetes, porque tenho diabetes, mas não dói nada dentro da barriga, nada, só fica ruim às vezes, mas doer, não. $\mathrm{E}$ cuidar da cabeça, mas a cabeça, acho que de velhice já tá birutando. E os dentes, eu queria antes arrumar, só que não deu por causa da diabetes.

Alguns acrescentavam a sua alimentação usual algumas orientaçóes médicas. Outros referiram diminuição do apetite e prazer pela comida - o que pode ser decorrente tanto da restriçáo ao consumo de alimentos que anteriormente lhes davam prazer quanto do processo de diminuiçấo do fluxo salivar pelo uso de determinados medicamentos. Distúrbios de gustação costumam ser observados em pacientes diabéticos e esse fato contribui negativamente para o tratamento, uma vez que o paladar diminuído pode resultar em hiperfagia, dificuldade em manter uma dieta adequada e, consequentemente, má regulação glicêmica ${ }^{15}$. A perda progressiva dos elementos dentais e o uso de próteses totais, como o que foi observado nos sujeitos deste relato de pesquisa, também podem estar relacionados à perda de apetite entre indivíduos idosos, uma vez que a diminuição da capacidade mastigatória leva à escolha de alimentos de baixa consistência e valor nutricional ${ }^{16}$.

Foi observada a compreensão de que HAS e DM são doenças crônicas e que é necessário controlá-las para evitar outros transtornos decorrentes dela:

P6: $A$ diabetes não cura, a gente sabe. A diabetes, controla ela, mas não cura. E a diabetes traz muitos problemas pra gente...

$\mathrm{O}$ autocuidado em saúde também prevê a adesão à terapêutica farmacológica, quando necessária. De maneira geral, os entrevistados utilizavam corretamente os medicamentos indicados e sabiam informar qual era sua finalidade, compreendendo a importância de cada fármaco no tratamento. Ao serem indagados a respeito de alterações, como a sensação de boca seca, sete entrevistados afirmaram que às vezes a sentiam, principalmente à noite. Alguns sujeitos relacionavam a sensação de boca seca com a respiração bucal, outros a associavam ao diabetes e à descompensação da hipertensão arterial, assim como houve associaçáo com o uso de medicamentos: 
P8: Eu desconfio que seja de algum comprimido que eu tomo e não está me fazendo muito bem. Agora não sei qual deles, porque eu tomo para o colesterol e para o coração, esses aí não é, mas para mim é a Amitriptilina, que eu uso pra dormir.

Essas alteraçóes bucais comuns do próprio processo de envelhecimento podem ser intensificadas pelo descontrole glicêmico do diabetes ${ }^{6}$. A hipossalivação - relatada por $10 \%$ a $30 \%$ dos diabéticos no país - pode estar relacionada à desidrataçáo decorrente da hiperglicemia e poliúria (eliminação excessiva de urina). Além disso, o uso de fármacos com ação anticolinérgica pode contribuir para reduzir a produção e o fluxo salivar. A deste, por sua vez, associado a alterações na composição da saliva e à hiperglicemia, resulta em uma maior susceptibilidade a infecçóes orais, sendo que, entre as patologias bucais mais recorrentes no DM não controlado, destaca-se a candidíase oral ${ }^{15}$.

A polifarmácia foi evidenciada, indicando possíveis interaçôes medicamentosas que potencializem o risco de agravos:

P3: Tomo remédio, o cálcio, porque meus ossos não são muito bons, já quebrei quatro costelas. Tomo remédio para controlar a pressão, tomo pra controlar o diabetes. Um pra não deixar a próstata crescer. E tomo Fenobarbital, porque eu tenho uma calcificação no cérebro.

P7: Eu tomo Omeprazol de manhã, dois em jejum, pra não atacar o estômago. Como eu tava tendo náusea de manhã, o gastro mandou eu tomar dois antes da janta também. Tomo Sinvastatina pro colesterol, parece, um à noite. Propanolol, dois por dia pra pressáo, e Dipirona. Paracetamol, a médica endócrino disse que não posso nem pensar por causa da hepatite C. Então agora eu tô tomando mais a Dipirona, só quando a dor é forte. Tomo de noite o Diazepan, pra dormir, acalmar um pouco. Aquele pra depressão, não sei o nome, "Protamezina"... Teria que tomar também Domperidona pro estômago, mas como tem que comprar, né, aí às vezes não tenho dinheiro, então não compro. Fluoxetina pra depressão.

Grande parte dos entrevistados relatou fazer uso de antidepressivos como Fluoxetina e Amitriptilina, anticonvulsivantes como Fenobarbital e Diazepam, além de anti-histamínicos como Prometazina, diuréticos e anti-hipertensivos, sendo que todos têm efeitos adversos ligados à hipossalivação e xerostomia ${ }^{17}$. A prevalência desses problemas durante a noite, como relatado pelos sujeitos da pesquisa, pode estar associada tanto à redução fisiológica do fluxo salivar no período do sono como ao uso de antidepressivos antes de dormir. De maneira geral, os entrevistados lidavam com o problema bebendo água. Também foi relatado o uso de gengibre para aliviar a sensação de boca seca.

Diante da tendência recente de novos arranjos familiares e aumento dos domicílios unipessoais, não deve ser desconsiderada a importância do apoio familiar, corresidente ou não, no bem-estar e qualidade de vida dos idosos, visto que nessa fase da vida tendem a uma maior susceptibilidade a problemas de saúde e insuficiência de uma rede de apoio ${ }^{18}$. Embora sejam idosos que morem sozinhos, é observada a participação da família como autocuidado apoiado:

P6: A minha filha, ela tem tanto cuidado comigo, ela diz: a mãe não pode tomar mais, não pode tomar errado, se cai um comprimido no chão que a mãe não sabe qual foi, bota na lata do lixo. Esse aqui é o da diabetes, eu tomo de noite, ela desenhou uma lua. Ela não deixa eu pegar remédio assim, tem medo que eu troque. Mas a minha cabeça tá boa ainda.

Ao serem questionados se havia algum cuidado especial para controle da hipertensão e do diabetes, foi frequente a menção à importância dos exercícios físicos e hábitos saudáveis visando a manter a autonomia, inclusive aproveitando recursos comunitários disponíveis no território.

A saúde bucal não está dissociada da saúde geral, sofrendo a influência da presença de doenças sistêmicas, tais como a hipertensão e o diabetes. A boa condiçâo de saúde bucal na terceira idade é um fator indispensável para o envelhecimento ativo e saudável, todavia, sua importância ainda não é devidamente reconhecida 5 .

\section{CONCLUSÕES}

Foi evidenciado que os idosos que vivem sozinhos e demonstram autonomia exercitam seu autocuidado em saúde bucal. Eles expressaram que assimilaram diversas orientações de saúde a sua rotina diária, adaptando os hábitos às novas necessidades, tendo em vista a presença de condições crônicas. Da mesma forma, expressaram compreender a vinculação entre determinadas terapêuticas farmacológicas e os agravos que lhes causam incômodo.

Identificamos a importância do vínculo com os profissionais de saúde e da adesão às terapêuticas propostas para manutenção da autonomia no cuidado em saúde bucal. Para tanto, é necessário que as equipes de saúde conheçam a realidade de vida de seus usuários e que o serviço de atenção primária preste informações que levem em consideraçáo o contexto social e cultural das pessoas atendidas. Com isso, poderão qualificar a atenção prestada a partir da identificação de potencialidades 
na promoção de saúde, bem como na prevenção de atitudes que dificultem a adesão a hábitos mais saudáveis. Assim, espera-se que as mudanças prescritas no estilo de vida façam sentido e sejam adotadas de maneira efetiva pelos idosos.

Foi identificada uma dinamicidade insuficiente do sistema de informaçóes para manter os respectivos registros atualizados, e, com isso, a agilidade na prestação do cuidado necessário a seus usuários pode ser prejudicada. Esse fato resultou em uma limitação do estudo, visto que o universo inicialmente previsto sofreu uma redução significativa no número de participantes ao aplicarmos a simultaneidade entre hipertensão e diabetes, inicialmente identificada via sistema de registro local, como critério de inclusão de participantes. Esperamos que a apropriação desta pesquisa pelas equipes de saúde possa estimulá-las a promover atualização periódica de seus registros.

A proposta deste estudo foi alcançada na medida em que conhecemos como idosos hipertensos e diabéticos que vivem sozinhos promovem seu autocuidado em saúde bucal, provendo informaçóes que poderão contribuir para o planejamento de açóes em saúde, atualização de fluxos e rotinas e possíveis mudanças de condutas e aprimoramento de serviços para melhorar a atenção à saúde desse grupo populacional. Consideramos que a população idosa que vive sozinha demonstra autonomia que deve ser apoiada por uma rede social e os serviços de saúde à qual está vinculada. Conhecer como cuidam de si, explicam seus problemas e buscam soluçóes permite a ampliação do cuidado em saúde, incluindo a identificaçáo de estreitamentos na produção de cuidado que devem ser examinados e superados.

Acreditamos que seria importante dar continuidade a este estudo agregando a avaliação clínica em saúde bucal com a finalidade de comparar a percepção autorreferida com a realidade clinicamente evidenciada.

\section{AGRADECIMENTOS}

Agradecemos aos participantes, por terem disponibilizado seu tempo e vivência para viabilizar esta produção; aos agentes comunitários de saúde, pelo auxílio na identificação de possíveis entrevistados; ao responsável pela avaliação e monitoramento da unidade de saúde Vila Floresta, por viabilizar o acesso ao sistema de informaçóes; e a Natália V. S. Schmitz, pela parceria na elaboraçáo do projeto de pesquisa e coleta dos dados. O agradecimento se estende igualmente ao Ministério da Saúde pela bolsa-residência, a qual permitiu dedicação exclusiva durante o período de residência.

\section{Referências}

1. Brasil. Instituto Brasileiro de Geografia e Estatística. Perfil dos idosos responsáveis pelos domicílios. 25 jul. 2002 [acesso em 2016 nov. 28]. Disponível em: http://bit.ly/2gaZOxt

2. Camargos MCS, Rodrigues RN, Machado CJ. Idoso, família e domicílio: uma revisão narrativa sobre a decisão de morar sozinho. Rev bras Est Pop. 2011 [acesso em 2014 jul. 1];28(1):217-30. Disponível em: http://bit. ly/2gyxSVc

3. Ramos JLC, Menezes MR, Meira EC. Idosos que moram sozinhos: desafios e potencialidades do cotidiano. Rev baiana enferm. 2010 [acesso em 2014 set. 1];24(1-3):4354. Disponível em: http://bit.ly/2gohJP1

4. Brasil. Rede Interagencial de Informaçóes para a Saúde. Indicadores e Dados Básicos - Brasil - 2012 [acesso em 2014 nov. 1]. Disponível em: http://bit.ly/1PoaWyc

5. Simões ACA, Oliveira RS, Carvalho DM. O envelhecimento no contexto da odontologia. Rev Triângulo. 2009 [acesso em 2014 ago. 1];2(1):43-52. Disponível em: http://bit.ly/2fIOTuz

6. Brasil. Ministério da Saúde. Secretaria de Atenção à Saúde. Departamento de Atenção Básica. Projeto SBBrasil 2010: Pesquisa Nacional de Saúde Bucal Resultados Principais. Brasília, DF: Ministério da Saúde; 2011 [acesso em 2015 out. 1]. Disponível em: http://bit. ly/2gOVISr

7. Khader YS, Dauod AS, El-Qaderi SS, Alkafajei A, Batayha WQ. Periodontal status of diabetics compared with nondiabetics: a metaanalysis. J Diabetes Complications. 2006 [acesso em 2014 nov. 1];20(1):5968. Disponível em: http://bit.ly/2ftb4X1

8. Lindhe J, Jang NP, Karring T. Tratado de periodontia clínica e implantodontia oral. 5a ed. Rio de Janeiro: Guanabara; 2010.

9. Brasil. Ministério da Saúde. Secretaria de Atenção à Saúde. Departamento de Atenção Básica. Caderno de Atenção Básica 37 - Estratégias para o cuidado da pessoa com doença crônica: hipertensão arterial sistêmica. Brasília, DF; 2013 [acesso em 2014 nov. 1]. Disponível em: http://bit.ly/20Dly7i

10. Wannmacher L, Ferreira MBC. Farmacologia clínica para dentistas. 3a ed. Rio de Janeiro: Guanabara Koogan; 2007

11. Silva ACS, Santos I. Promoção do autocuidado de idosos para o envelhecer saudável: aplicação da teoria de Nola Pender. Texto contexto - enferm. 2010 [acesso em 2014 out. 1];19(4):745-53. Disponível em: http://bit. ly/2gbeGvQ

12. Minayo MCS. O desafio do conhecimento: pesquisa qualitativa em saúde. 11a ed. São Paulo: Hucitec; 2008.

13. Haikal DS, Paulo AMB, Martins AMEBL, Moreira AN, Ferreira EF. Autopercepção da saúde bucal e impacto na qualidade de vida do idoso: uma abordagem 
quanti-qualitativa. Ciênc saúde coletiva. 2011 [acesso em 2015 nov. 1];16(7):3317-29. Disponível em: http:// bit.ly/2gyOdJv

14. Bez AS. Resiliência e sua relação com mudança na autopercepção de saúde bucal em idosos independentes do sul do Brasil [dissertação]. Porto Alegre: Universidade Federal do Rio Grande do Sul; 2013 [acesso em 2014 jul. 1]. Disponível em: http://bit.ly/2gCimVI

15. Brasil. Ministério da Saúde. Secretaria de Atenção à Saúde. Departamento de Atenção Básica. Caderno de Atenção Básica 36 - Estratégias para o cuidado da pessoa com doença crônica: Diabetes Mellitus. Brasília, DF; 2013 [acesso em 2016 nov. 28]. Disponível em: http://bit.ly/2gOPqNl
16. Campos MTFS, Monteiro JBR, Ornelas APRC. Fatores que afetam o consumo alimentar e a nutrição do idoso. Rev Nutr. 2000 [acesso em 2015 nov, 1];13(3):157-65. Disponível em: http://bit.ly/2gok1hb

17. Vidal ACC, Lima GA, Grinfeld S. Pacientes idosos: relação entre xerostomia e o uso de diuréticos, antidepressivos e antihipertensivos. IJD. 2004 [acesso em 2015 set. 1];3(1):330-35. Disponível em: http://bit.ly/2gbiGwA

18. Camargos MCS, Rodrigues RN. Idosos que vivem sozinhos: como eles enfrentam dificuldades de saúde. Anais do XVI Encontro Nacional de Estudos Populacionais; 2008. Caxambu: ABEP; 2008 [acesso em 2014 set. 1]. Disponível em: http://bit.ly/2gOUn8I

\section{Como citar este artigo:}

Lima P, Fajardo AP. Aspectos do autocuidado em saúde bucal de idosos hipertensos e diabéticos que vivem sozinhos. Rev. Aten. Saúde. 2016;14(50):56-62. 